This item was submitted to Loughborough's Research Repository by the author.

Items in Figshare are protected by copyright, with all rights reserved, unless otherwise indicated.

\title{
Coupling crystal plasticity and continuum damage mechanics for creep assessment in Cr-based power-plant steel
}

\section{PLEASE CITE THE PUBLISHED VERSION}

https://doi.org/10.1016/j.mechmat.2019.01.006

PUBLISHER

Crown copyright $@$ Elsevier BV

VERSION

AM (Accepted Manuscript)

\section{PUBLISHER STATEMENT}

This paper was accepted for publication in the journal Mechanics of Materials and the definitive published version is available at https://doi.org/10.1016/j.mechmat.2019.01.006.

\section{LICENCE}

CC BY-NC-ND 4.0

\section{REPOSITORY RECORD}

Zhao, Nailong, Anish Roy, Weizhe Wang, Liguo Zhao, and Vadim Silberschmidt. 2019. "Coupling Crystal Plasticity and Continuum Damage Mechanics for Creep Assessment in Cr-based Power-plant Steel". figshare. https://hdl.handle.net/2134/36654. 


\title{
Coupling crystal plasticity and continuum damage mechanics for creep assessment in Cr-based power-plant steel
}

\author{
Nailong Zhao ${ }^{1,2}$, Anish Roy ${ }^{2}$, Weizhe Wang ${ }^{1}$, Liguo Zhao ${ }^{2}$ and Vadim V. Silberschmidt ${ }^{2}$ \\ ${ }^{1}$ Key Laboratory of Power Machinery and Engineering, School of Mechanical Engineering, Shanghai \\ Jiao Tong University, Shanghai, 200240, P.R. China \\ ${ }^{2}$ Wolfson School of Mechanical, Electrical and Manufacturing Engineering, Loughborough \\ University, Loughborough, LE11 3TU, UK
}

Key-Words: Crystal Plasticity; Continuum Damage; Creep; Finite Element; Power plant.

\begin{abstract}
To improve the design and safety of power plant components, long-term hightemperature creep behaviour of a power-plant material, such as Cr-based alloy, should be assessed. Prior studies indicate that power-plant components undergo material degradation and premature failure by nucleation, growth and coalescence of microvoids as a result of creep damage. In classical crystal-plasticity-based models, a flow rule and a hardening law do not account for global stiffness degradation of materials due to evolving microvoids, having a significant influence on material behaviour, especially under large deformations. In this study, a crystal-plasticity scheme coupled with an appropriate continuum damage model is developed to capture the anisotropic creep-damage effect on the overall deformation behaviour of Cr-based power-plant steel. Numerical simulations show that the developed approach can characterize creep deformation of the material exposed to a range of stress levels and temperatures under consideration of stiffness degradation under large deformation.
\end{abstract}




\section{Introduction}

Power-plant components are commonly exposed to time-varying mechanical and thermal loads at elevated temperatures; as a result, effects of multiaxial creep deformation should be fully assessed to ensure safe operation of the component during its lifetime. Extensive research has been conducted to characterize creep deformation of power-plant materials using macroscopic continuum-damage models (Cui and Wang, 2014; Jiang et al., 2015; JianPing et al., 2003; Wichtmann, n.d.; Xu, 2007), which account for progressive degradation of mechanical properties caused by irreversible microstructural changes during the deformation process. Creep damage, accompanied by nucleation, growth and coalescence of microvoids, is closely related to the microstructure of materials. However, macroscopic constitutive models do not account for the underlying microstructure of the material (for example, activation of slip systems and void nucleation at grain boundaries are ignored). As a result, such models have inherent drawbacks in their predictive capability.

For crystalline materials, it is important to incorporate the effect of underlying microstructure and associated micromechanics into a continuum constitutive description of finite-strain plasticity. A popular physics-based crystal plasticity (CP) modelling framework was successfully used to account for important micromechanical features of plastic flow at the scale of single grains (Roters et al., 2010). To study the material failure, CP may be coupled with an appropriate damage-mechanics model (Ekh et al., 2004; Feng et al., 2004; Hu et al., 2016; Kalnaus and Jiang, 2006; Potirniche et al., 2007). Most studies are still concerned with single-crystal materials, and often ignore a complex polycrystalline microstructure of real-life components. Luo et al. (Luo et al., 2009; Luo and Chattopadhyay, 2011) introduced a multiscale damage criterion incorporating $\mathrm{CP}$ at the microscale, which was suitable for predicting the initiation of fatigue cracks in components made of polycrystalline Al-based alloy. Anahid et al. (Anahid et al., 2011) developed a CP-based crack-nucleation model for polycrystalline microstructures considering room-temperature creep phenomenon and applied it to predict fatigue-crack nucleation in a dual-phase titanium alloy undergoing cyclic dwell loading. However, these models did not consider a post-crack-nucleation response of the material. For power-plant materials, such as polycrystalline Cr-based alloys, to the best of the authors' knowledge, no studies exist on modelling of creep-damage behaviour based on the $\mathrm{CP}$ theory, especially under consideration of stiffness degradation during all three stages of creep.

In this paper, a constitutive model coupling $\mathrm{CP}$ and continuum damage mechanics (CDM) 
was developed and implemented in a computational framework for assessing the creep behaviour of a Cr-based power-plant material. A second-order symmetric tensor was used in this model to describe the anisotropic creep damage. The damage-evolution equation was thermodynamically consistent, accounting for the variation of material stiffness with induced damage. Uniaxial creep tests at three temperatures were used to calibrate damage parameters. The coupled numerical model was implemented as a user material subroutine in the generalpurpose finite-element (FE) program Abaqus/Explicit (Abaqus, n.d.). A 3-D polycrystalline representative volume element (RVE) was generated to model the underlying microstructure of the material. A series of numerical simulations were conducted based on the RVE to demonstrate the capability of the coupled model in creep assessment of the material. 


\section{Constitutive Model}

Here, the constitutive model coupling CP with a CDM-based approach is presented. A material subroutine (VUMAT) was developed based on the work of Huang (Huang, 1991) and Busso (Busso et al., 2000; Meissonnier et al., 2001). The VUMAT allows element deletion in the FE analysis, indicating the nucleation and growth of microvoids in the polycrystalline material.

\subsection{Crystal plasticity}

Here, a brief summary of the crystal-plasticity modelling framework used to capture an anisotropic constitutive response due to the effect of grain morphology and crystallographic orientation is provided.

The deformation gradient $\mathbf{F}$ can be decomposed into elastic and plastic parts as,

$$
\mathbf{F}=\mathbf{F}^{e} \cdot \mathbf{F}^{p},
$$

where $\mathbf{F}^{p}$ is plastic deformation in an intermediate configuration, and $\mathbf{F}^{e}$ is the elastic component of the deformation gradient, which includes deformation by both stretching and rotation of the lattice. The velocity gradient, $\mathbf{L}$, in the current configuration is related to the deformation gradient by

$$
\mathbf{L}=\dot{\mathbf{F}} \mathbf{F}^{-1}=\mathbf{L}^{e}+\mathbf{L}^{p}
$$

where $\mathbf{L}^{e}=\dot{\mathbf{F}}^{e} \mathbf{F}^{e-1}$ and $\mathbf{L}^{p}=\mathbf{F}^{e} \dot{\mathbf{F}}^{p} \mathbf{F}^{p-1} \mathbf{F}^{e-1}$.

The inelastic deformation of a single crystal is assumed to be generated only from the crystalline slip. The plastic velocity gradient may be rewritten in terms of the slip strain rate, $\dot{\gamma}^{(\alpha)}$, the slip direction, $\mathbf{S}^{(\alpha)}$, and the normal to the slip plane, $\mathbf{m}^{(\alpha)}$, as

$$
\mathbf{L}^{p}=\sum_{\alpha} \dot{\gamma}^{(\alpha)} \mathbf{S}^{(\alpha)} \otimes \mathbf{m}^{(\alpha)},
$$

where $\alpha$ represents the slip system (ranging from 1 to 48 for this BCC material). The resolved shear stress is derived from the Cauchy stress tensor by

$$
\tau^{(\alpha)}=\boldsymbol{\sigma}:\left(\mathbf{S}^{(\alpha)} \otimes \mathbf{m}^{(\alpha)}\right)_{s y m} .
$$

where $\otimes$ is the dyadic product and sym is the symmetrical part of the tensor. The slip direction and the normal to the slip plane (as used above) are in the deformed configuration. The relationship between the slip systems in the deformed configuration and those in the reference configuration can be expressed as: 


$$
\left\{\begin{array}{l}
\mathbf{S}^{(\alpha)}=\mathbf{F}^{e} \mathbf{S}_{0}^{(\alpha)} \\
\mathbf{m}^{(\alpha)}=\mathbf{m}_{0}^{(\alpha)} \mathbf{F}^{e-1}
\end{array}\right.
$$

where $\mathbf{S}_{0}^{(\alpha)}$ and $\mathbf{m}_{0}^{(\alpha)}$ are the slip direction and slip plane normal, respectively, in the reference configuration for the slip system $\alpha$. The flow rule is assumed to follow a powerlaw relation (Huang, 1991),

$$
\dot{\gamma}^{(\alpha)}=\dot{\gamma}_{0}^{(\alpha)}\left|\frac{\tau^{(\alpha)}-\chi^{(\alpha)}}{g^{(\alpha)}}\right|^{n} \operatorname{sgn}\left(\tau^{(\alpha)}-\chi^{(\alpha)}\right)
$$

where $g^{(\alpha)}$ and $\chi^{(\alpha)}$ are the isotropic hardening and kinematic hardening, respectively, and sgn is the sign of the term. The hardening laws are presented as (Sinha and Ghosh, 2006),

$$
\begin{aligned}
& \dot{g}^{(\alpha)}=\sum_{\beta} h_{\alpha \beta} \dot{\gamma}^{\beta}, \\
& h_{\alpha \beta}=\left\{\begin{array}{c}
h(\gamma)=h_{0} \sec h^{2}\left|\frac{h_{0} \gamma}{\tau_{s}-\tau_{0}}\right|, \quad \alpha=\beta \\
q h(\gamma), \quad \begin{array}{r}
\alpha \neq \beta
\end{array},
\end{array}\right.
\end{aligned}
$$

and

$$
\dot{\chi}^{(\alpha)}=b \dot{\gamma}^{(\alpha)}-r \chi^{(\alpha)}\left|\dot{\gamma}^{(\alpha)}\right|,
$$

where $h_{\alpha \beta}$ are the isotropic hardening moduli. The variables $b$ and $r$ are the direct hardening and dynamic recovery coefficients, respectively, and $q$ is the latent hardening parameter.

The Cr-based alloy, X12CrMoWVNbN10-1-1, may be the most representative kind of ferrite-martensite steel in thermal power units. It has a body-centered cubic (BCC) crystalline structure with 12 main slip systems $(\{110\}<111>)$ and 36 secondary slip systems $(\{112\}$ $<111>$ and $\{123\}<111>$ ), which contribute to crystalline slip (Golden et al., 2014; Li et al., 2017). The cumulative shear strain on all slip systems was obtained by

$$
\gamma=\sum_{\alpha} \int_{0}^{t} \dot{\gamma}^{(\alpha)} \mid d t
$$

Material parameters in the CP model (shown in Table 1) are calibrated using experimental data obtained from isothermal strain-controlled cyclic tension and compression experimental tests at $550^{\circ} \mathrm{C}, 600^{\circ} \mathrm{C}$, and $625^{\circ} \mathrm{C}$, and further validated by isothermal strain-controlled test data with holding times. Details are available in a prior work of some of the authors (Zhao et al., 2017). 
Table 1 Material parameters in $\mathrm{CP}$ at different temperatures.

\begin{tabular}{c|c|c|c}
\hline Elastic modulus & $550^{\circ} \mathrm{C}$ & $600^{\circ} \mathrm{C}$ & $625^{\circ} \mathrm{C}$ \\
\hline$C_{11} / \mathrm{GPa}$ & 174 & 167.2 & 163.2 \\
\hline$C_{12} / \mathrm{GPa}$ & 146.2 & 140.5 & 138.4 \\
\hline$C_{44} / \mathrm{GPa}$ & 78 & 74.9 & 73.2 \\
\hline \multicolumn{2}{|c|}{ Material constants in flow rule } \\
\hline \multicolumn{2}{|c|}{0.001} \\
\hline
\end{tabular}

Material constants in self- and latent- isotropic hardening for the main slip systems

\begin{tabular}{c|c|c|c}
\hline$h_{0} / \mathrm{MPa}$ & 1420 & 1340 & 1200 \\
\hline$\tau_{s} / \mathrm{MPa}$ & 280 & 270 & 240 \\
\hline$\tau_{0} / \mathrm{MPa}$ & 160 & 150 & 135 \\
\hline$q$ & \multicolumn{3}{|c}{1} \\
\hline
\end{tabular}

Material constants in self- and latent- isotropic hardening for the secondary slip systems

\begin{tabular}{c|c|c|c}
\hline$h_{0} / \mathrm{MPa}$ & 7100 & 6700 & 6000 \\
\hline$\tau_{s} / \mathrm{MPa}$ & 1400 & 1350 & 1200 \\
\hline$\tau_{0} / \mathrm{MPa}$ & 800 & 750 & 675 \\
\hline$q$ & \multicolumn{3}{|c}{1} \\
\hline \multicolumn{1}{|c|}{ Material constants in kinematic hardening } \\
\hline$b / \mathrm{MPa}$ & 6150 & 5800 & 5450 \\
\hline$r$ & & 2 \\
\hline
\end{tabular}

\subsection{Continuum damage mechanics}

Since the pioneering work of Kachanov (Kachanov, 1958) and Rabotnov (Rabotnov, 1969), CDM has been extensively used in research. CDM attempts to represent the progressive mechanical degradation of materials subjected to imposed mechanical loads. It is well-known that creep is accompanied by nucleation, growth and coalescence of microvoids. The process of formation of microvoids occurs anisotropically in the material volume. At the scale of individual grains, in addition to material texture, damage-induced anisotropy must be accounted for. In this regard, a second-order symmetric tensor may be used to represent 
geometric properties of a given state of microvoids in the continuum (Qi and Bertram, 1998, 1999).

According to the effective-stress concept of CDM, mechanical behaviour of the material considering damage may be described by a constitutive equation, where the stress tensor is replaced by an effective stress tensor, $\tilde{\boldsymbol{\sigma}}$, which accounts for the current state of damage in the material. The effective stress in the present model is defined as (Lemaitre and Chaboche, 1994)

$$
\tilde{\boldsymbol{\sigma}}=(\mathbf{I}-\mathbf{D})^{-1 / 2} \cdot \boldsymbol{\sigma} \cdot(\mathbf{I}-\mathbf{D})^{-1 / 2},
$$

where $\boldsymbol{\sigma}, \mathbf{I}$ and $\mathbf{D}$ denote the stress tensor, the identity tensor of rank two and (anisotropic) damage tensor, respectively.

Considering the influence of damage (material stiffness degradation) on material deformation, the resolved shear stress (Eq. (4)) and isotropic hardening variable in CP are rewritten as (Feng et al., 2004):

$$
\tilde{\tau}^{(\alpha)}=\tilde{\boldsymbol{\sigma}}:\left(\mathbf{S}^{(\alpha)} \otimes \mathbf{m}^{(\alpha)}\right)_{s y m},
$$

and

$$
\tilde{g}^{(\alpha)}=\left((\mathbf{I}-\mathbf{D}):\left(\mathbf{S}^{(\alpha)} \otimes \mathbf{m}^{(\alpha)}\right)_{s y m}\right) g^{(\alpha)} .
$$

Accordingly, the flow rule (Eq. (6)) is redefined as

$$
\dot{\gamma}^{(\alpha)}=\dot{\gamma}_{0}^{(\alpha)}\left|\frac{\tilde{\tau}^{(\alpha)}-\chi^{(\alpha)}}{\tilde{g}^{(\alpha)}}\right|^{n} \operatorname{sgn}\left(\tilde{\tau}^{(\alpha)}-\chi^{(\alpha)}\right) \cdot
$$

Here, both isotropic hardening and the slip in individual slip systems are coupled to account for damage. Thus, damage is expected to influence the overall material deformation, especially at the secondary and tertiary creep stages (where damage is significant).

\subsection{Damage assessment and evolution}

Damage in the material is caused by progressive irreversible changes in its internal structure during deformation, which leads to a reduction of stiffness. Creep damage, accompanied by the nucleation, growth and coalescence of microcracks and microvoids, has anisotropic characteristics in a multiaxial stress state, which should be accounted for in the damage assessment.

For a process undergoing creep, damage may be expressed as (JianPing et al., 2003; Lemaitre and Chaboche, 1994) 


$$
D_{i j}=1-\left(\frac{\dot{\varepsilon}_{i j}^{\text {II }}}{\dot{\varepsilon}_{i j}^{I I I}}\right)^{1 / n},
$$

where $D_{i j}$ are components of the damage tensor $\mathbf{D}, \dot{\varepsilon}_{i j}^{\mathrm{II}}$ and $\dot{\varepsilon}_{i j}^{\mathrm{II}}$ are the secondary and tertiary creep rates, respectively, which are measured as functions of time on the creep curve, and $n^{\prime}$ is a material parameter that needs to be calibrated.

A thermodynamically consistent creep damage evolution as proposed by Lemaitre and Chaboche (Lemaitre and Chaboche, 1994) is adopted here, which is known to be ideally suited for Cr-based alloys (Jing et al., 2003). An incremental change in damage ( $d \mathbf{D}$ ) is represented by

$$
d \mathbf{D}=\frac{1}{(\mathbf{I}-\mathbf{D})^{\beta}} \cdot\left(\frac{\tilde{\sigma}}{\lambda}\right)^{m} d t
$$

where $\beta, \lambda, m$ are material parameters to be determined and $d t$ is a time increment. The term $(\mathbf{I}-\mathbf{D})^{\beta}$ implies a tensor power series.

To determine parameters in CDM, uniaxial creep experiments on standard specimen were performed. Experimental data were assessed at temperatures of $550^{\circ} \mathrm{C}, 600^{\circ} \mathrm{C}$ and $625^{\circ} \mathrm{C}$ (these are common operating temperatures for power-plant materials), with three stress levels considered for each temperature. Further details of the experimental procedure are available in (Wang et al., 2015).

Assuming an initial damage-free state (i.e. $D=0$ at $t=0$ ) in Eq. (16), the relation for uniaxial creep damage can be obtained in terms of stress and time via integration:

$$
D=1-\left[1-(1+\beta)\left(\frac{\tilde{\sigma}}{\lambda}\right)^{m} t\right]^{\frac{1}{1+\beta}} .
$$

By defining a rupture time $\left(t_{c}\right)$, corresponding to a state of critical damage $\left(D_{c}\right)$, the following relation can be obtained:

$$
t_{c}=\frac{1}{1+\beta}\left[1-\left(1-D_{c}\right)^{1+\beta}\right]\left(\frac{\tilde{\sigma}}{\lambda}\right)^{-m} \text {. }
$$

For most metallic materials, $\beta$ ranges from 5 to 15 and $D_{c}$ from 0.3 to 0.9 . Hence, $\left(1-D_{c}\right)^{1+\beta}$ may be ignored. Thus, $t_{c}$ and $D$ may be simplified as

$$
t_{c}=\frac{1}{1+\beta}\left(\frac{\tilde{\sigma}}{\lambda}\right)^{-m}
$$

and

$$
D=1-\left[1-t / t_{c}\right]^{\frac{1}{1+\beta}} .
$$


At each temperature, the initial values of $m$ and $\lambda$ can be calculated by using two creep curves (at two stress levels- $\sigma_{1}$ and $\sigma_{2}$ ) with $t_{c}$ as (Shi and Yang, 2004),

$$
r=\frac{\ln t_{c 1}-\ln t_{c 2}}{\ln \sigma_{2}-\ln \sigma_{1}}
$$

and

$$
\lambda=\frac{\tilde{\sigma}_{1}\left[t_{c 1}(1+\beta)\right]^{1 / m}+\tilde{\sigma}_{2}\left[t_{c 2}(1+\beta)\right]^{1 / m}}{2} .
$$

After determination of initial values, the parameters are optimized based on experimental data for each temperature using the least-square method (Fig. 1); Table 2 lists the optimised values. We observe that $\beta$ increases with temperature, while $\lambda$ shows an inverse effect. To determine the parameters at other temperatures an appropriate interpolation technique may be used.

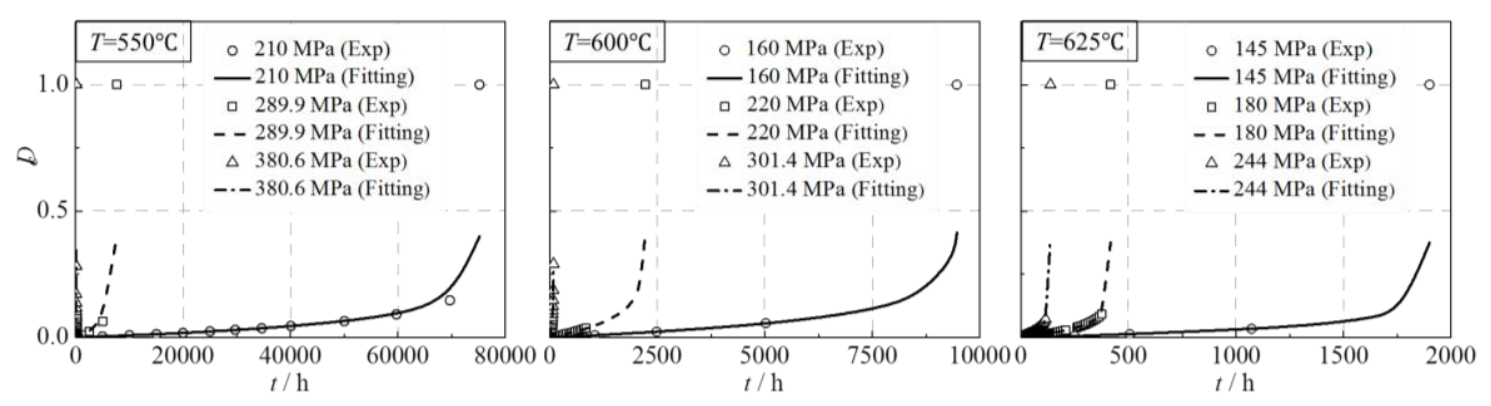

Fig. 1 Calibration of damage parameters at three temperatures.

Table 2 Material parameters in CDM at different temperatures.

\begin{tabular}{c|c|c|c}
\hline Parameters & $550^{\circ} \mathrm{C}$ & $600^{\circ} \mathrm{C}$ & $625^{\circ} \mathrm{C}$ \\
\hline$\beta$ & 15.47 & 16.27 & 22.95 \\
\hline$\lambda$ & 4800.47 & 2510.88 & 2150.59 \\
\hline$m$ & \multicolumn{3}{|c}{7.15} \\
\hline
\end{tabular}

In the optimization process, the first and secondary stages of creep and the transition between the secondary and tertiary stage play a significant role. During the (final) tertiary stage, coalescence of microvoids occurs extensively leading to a rapid rise in damage. However, the mechanism of void coalescence is beyond the scope of the current damage model. Hence, we do not attempt to model/predict damage beyond $D=0.4$. It is worth noting that the tertiarty stage of creep is very short compared to the other two stages. From a practical standpoint, the initiation of tertiary stage and failure are often treated to be identical. 
Based on this consideration, the critical damage value $\left(D_{c}\right)$ was set as 0.4 in the FE analysis, which implies element deletion when one of its damage components reaches or exceeds 0.4. Since, the damage level increases rapidly after this value, the simulated results are not representative of the experimental tests. After deletion, the element will be subjected to zero stresses and strain increments (Abaqus, n.d.), essentially implying the generation of a microvoid at the location. 


\section{Finite-element model}

3-D polycrystalline FE models were developed in this study to demonstrate the capability of the coupled model for creep-damage assessment. An RVE based approach (Kovač and Cizelj, 2005) was implemented to account for the underlying polycrystallinity of the component. By using this approach, a homogenized mesoscale stress-strain response can be obtained (Zhang et al., 2013; 2015).

\subsection{3-D polycrystalline FE model}

A cubic RVE of a side of $1.5 \mathrm{~mm}$, containing 27 grains was generated with the Voronoi tessellation technique using a freely available software (Quey et al., 2011), as shown in Fig. 2 (a) (together with a FE mesh). The average grain size was determined by optical microscopy studies of $12 \% \mathrm{Cr}$ steel (Dong et al., 2013; Yaso et al., 2009). Temperature-dependent physical properties of the material are available in (Wang, 2014). A set of random crystallographic orientations was assigned to each grain, with the inverse pole figure shown in Fig. 2 (b). Tetrahedron elements (C3D4) available in Abaqus (Abaqus, n.d.) were used in the FE analysis. A mesh-sensitivity study was carried out for the polycrystalline model, with three sets of elements: 989, 3204 and 5536. It is imperative to assess the local stresses and the damage parameter in mesh convergence studies, as a substantial variation in the local stresses would inevitably affect the onset of creep damage in the material. Results obtained from the mesh convergence study show acceptable convergence for the 3204-element mesh.

Periodic boundary conditions were imposed on the four lateral faces $\left(\mathrm{X}_{0}, \mathrm{X}_{1}, \mathrm{Z}_{0}\right.$ and $\left.\mathrm{Z}_{1}\right)$ as shown in Fig. 2 (c). This effectively treats the domain boundary as grain boundaries of the chosen polycrystalline ensemble. The bottom surface $\left(\mathrm{Y}_{0}\right)$ was fixed in the Y-direction. Tensile stress was applied on the top face in the positive $\mathrm{y}$-direction $\left(\mathrm{Y}_{1}\right)$ to simulate the uniaxial stress-controlled loading condition. To obtain the macroscopic mechanical response of the material, homogenized strain $\left(\bar{\varepsilon}_{i j}\right)$ and damage $\left(\bar{D}_{i j}\right)$ responses were evaluated for the RVE volume:

$$
\bar{\varepsilon}_{i j}=\frac{1}{V} \int_{V} \varepsilon_{i j} d V
$$

and

$$
\bar{D}_{i j}=\frac{1}{V} \int_{V} D_{i j} d V
$$




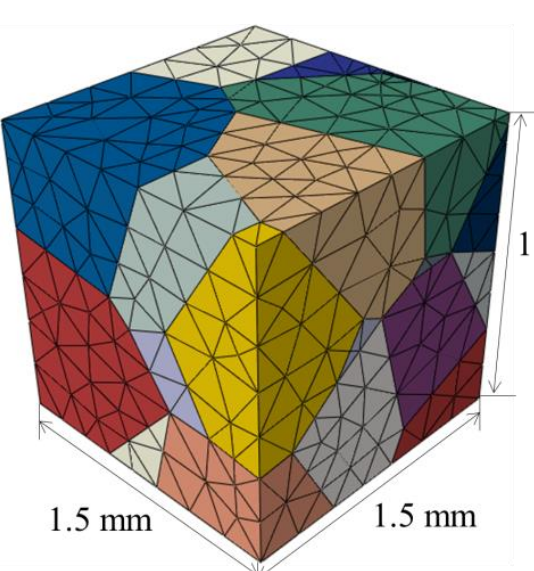

(a)

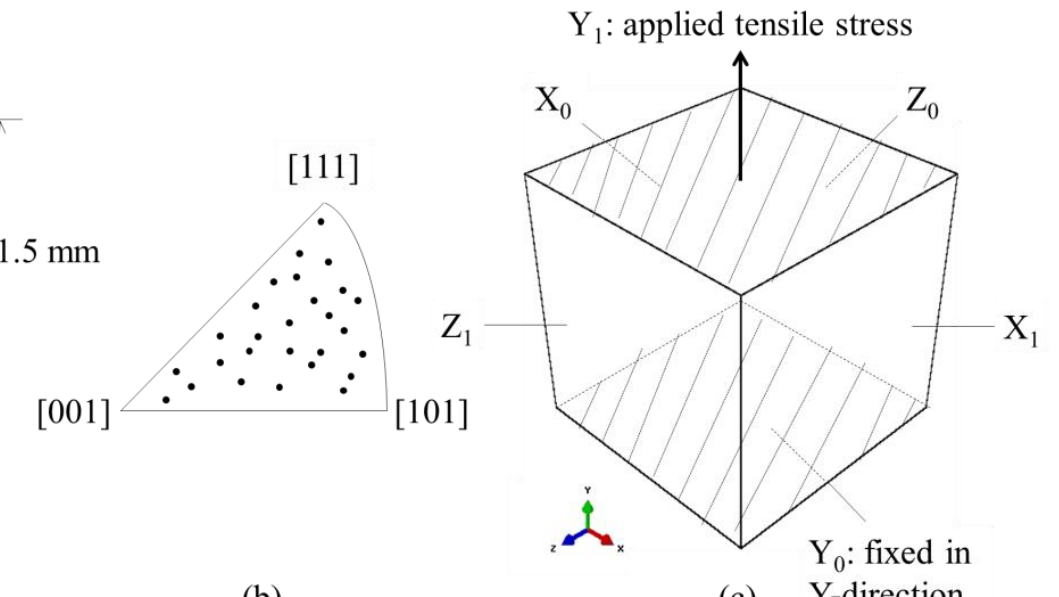

(b)

(c) Y-direction

Fig. 2 (a) Geometry and FE mesh of 3-D 27-grains RVE model (colours designate crystallographic orientation); (b) inverse pole figure for grains in RVE model; (c) schematic of imposed boundary conditions.

\subsection{Effect of grain orientation}

To assess the possible influence of the chosen random crystallographic orientations on the obtained macroscopic results, an alternative set of (randomly generated) orientations was assigned to the polycrystalline structure in the RVE with identical boundary conditions. A comparison of global strain for varying temperature and stress states is shown in Fig. 3. The random crystallographic orientations have almost no effect on the strain response during the first and secondary stages of creep, with minor differences during the tertiary stage. The maximum difference in strain was $<5.5 \%$ for the three loading conditions, which implies that the effect of chosen (random) grain orientations on the global response was limited.
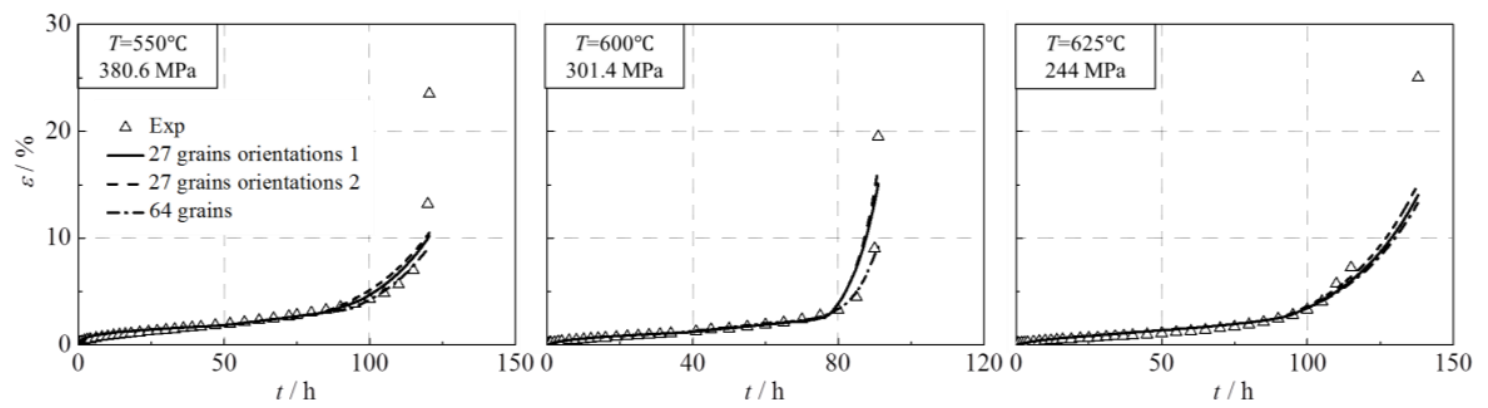

Fig. 3 Strain comparison for different sets of orientations and grain numbers of RVE model.

\subsection{Effect of number of grains in RVE}

The chosen number of grains in the RVE model needs to be sufficiently large to represent the entire material volume. To study this an RVE of $2 \times 2 \times 2 \mathrm{~mm}^{3}$ size with 64 grains of the 
same average size with random crystalline orientations was generated employing the Voronoi tessellation technique; Figure 4 (a) shows the corresponding geometry and mesh. The imposed (periodic) boundary conditions were the same as in the model described in Section 3.1. Because of long simulation times, calculations were carried out at the maximum imposed stress with short creep fracture time for each temperature; the comparison of global strain is shown in Fig. 3. During the first and secondary stages of creep, there were almost no differences between the results for 27- and 64-grain FE models. At the tertiary stage, the creep strain of the 64-grain FE model was found to be slightly smaller than that for the 27-grain FE model at the stress of $301.4 \mathrm{MPa}$ at $600^{\circ} \mathrm{C}$; these differences are not significant. Therefore, $\mathrm{FE}$ analysis was performed with the RVE with 27 grains meshed with 3204 elements to evaluate global and local creep deformations for all other loading conditions for numerical and computational efficiency.

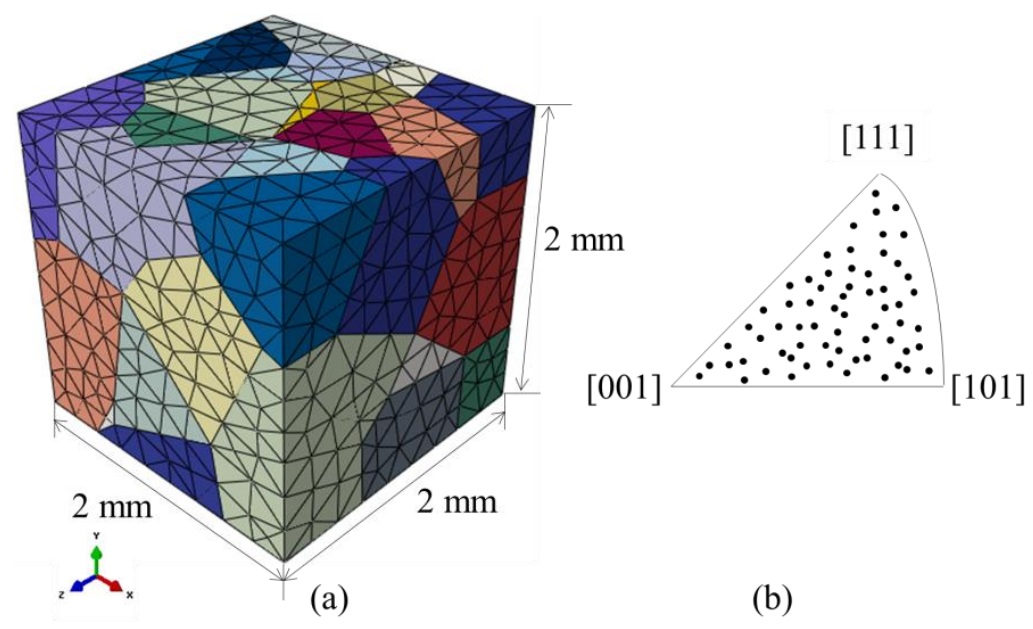

Fig. 4 (a) Geometry and FE mesh of a 3-D 64-grains polycrystalline RVE model (colours designate crystallographic orientation); (b) inverse pole figure for grains in model. 


\section{Results and discussion}

In this section, numerical results obtained with the coupled $\mathrm{CP}$ and $\mathrm{CDM}$ model are presented and some of the critical observations are discussed. In particular, the focus is on macro- and microscale-variables used in the model and their effect on prediction of creep in the material.

\subsection{Prediction of creep deformation}

First, creep deformation was studied using the developed coupled model at the macroscale. Figure 5 provides a comparison of the numerical results with the experimental data at different stress levels and temperatures. The strain-time curves obtained with the coupled numerical model capture the three stages of creep deformation reasonably well, except for the last measured state of maximum strain for each case. This was due to the lack of accounting of the mechanism of microvoids coalescence in the damage model; as a result, strain prediction for the tertiary creep was not accurate. Interestingly, a rapid strain increase during tertiary creep was successfully captured.

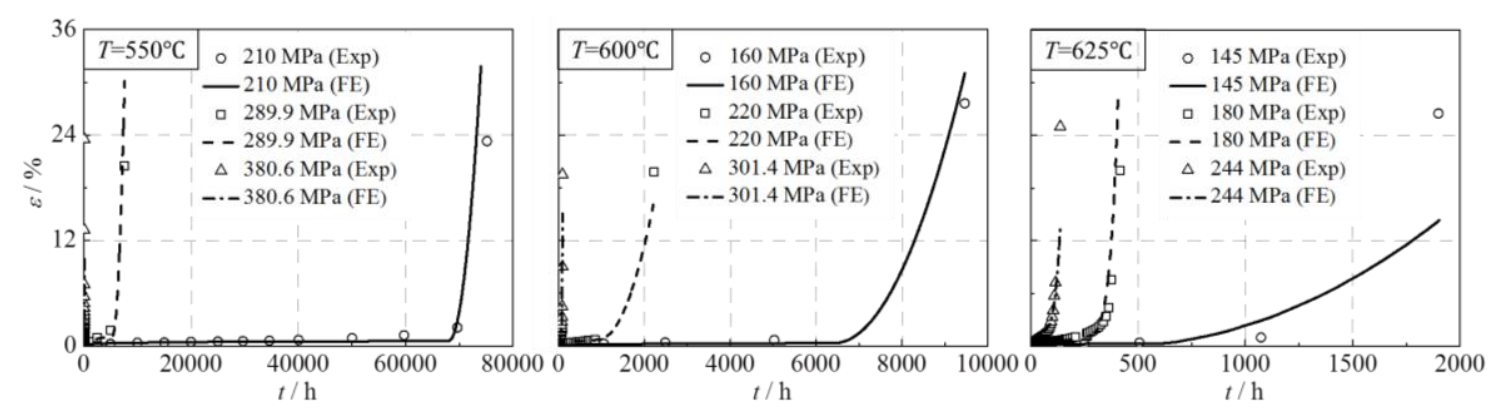

Fig. 5 Comparison of simulated and measured strains at different stresses at three temperatures.

To assess the effectiveness of the proposed coupled model accounting for damage, creep curves calculated with the coupled model and the pure CP model (without damage) were compared for the maximum stress at each temperature (Fig. 6). It was found that the creep rate obtained from the coupled model was always higher than that calculated with the $\mathrm{CP}$ model. Though the differences were small at the early stages of creep, the difference became larger with increasing damage accumulation in the material. The coupled model with damage was capable of capturing the complete creep behaviour till rupture, thus such an approach may be used in prediction of material creep lifetime. Without the consideration of damage, the 
strain kept increasing at a steady rate (as there was no reduction in material stiffness) after the initial rapid rise (the first stage of creep). This leads to a deviation at secondary and tertiary stages of creep.
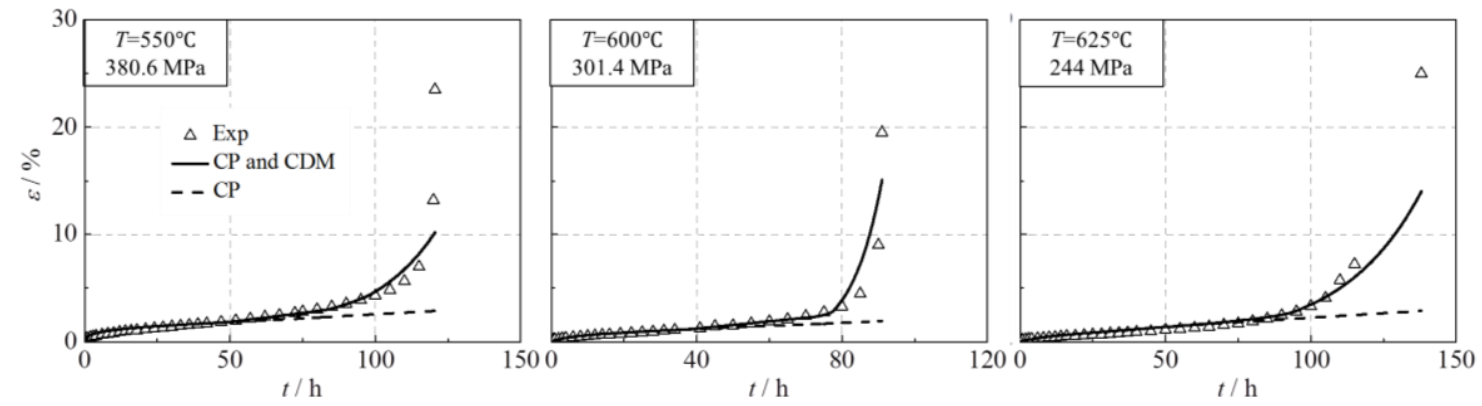

Fig. 6 Comparison of creep strain predictions with coupled model and CP model, for three loading cases.

\subsection{Damage evolution}

Damage plays a significant role in prediction of creep deformation, especially with increasing loading time (Fig. 6). Under uniaxial tension the stress was applied in the positive Y-direction (shown in Fig. 2 (c)); hence the normal damage component in this direction $\left(D_{22}\right)$ was much larger than the other normal components $\left(D_{11}, D_{33}\right)$. The variation of average damage components $\left(D_{11}, D_{22}\right.$ and $\left.D_{33}\right)$ in the material is shown in Fig. 7. Here, the values presented were obtained by averaging the value of all elements in the mesh including the deleted elements; this was done by assigning a value of 1 to those elements. Generally, the damage parameter quickly reaches this level once the value exceeds 0.4 -the critical value for element deletion. The results show that the average values of $D_{11}$ and $D_{33}$ were nearly identical, and approximately three orders of magnitude lower than that of $D_{22}$. The damage corresponding to the direction of uniaxial tension $\left(D_{22}\right)$ exhibited the highest magnitude, as expected. Thus, the developed model captured the anisotropic damage evolution in addition to the elastic-plastic crystalline anisotropy. 


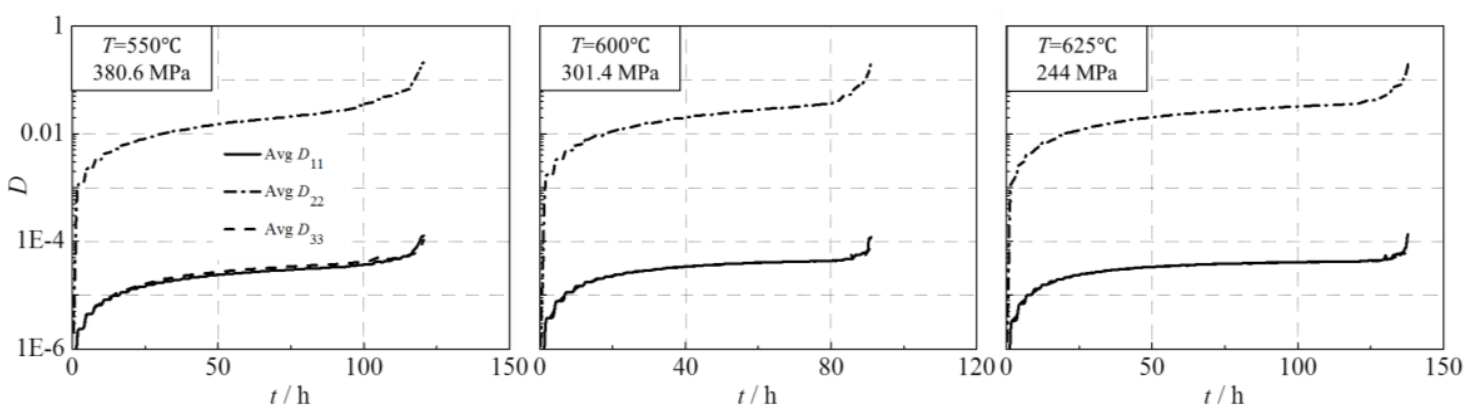

Fig. 7 Variation of average damage components at the maximum stress at three temperatures.

Evolution of the $D_{22}$ damage variable at the three loading cases are shown in Fig. 8. Three relevant moments of time, corresponding to (i) the time just before deletion of the first element, (ii) the time when the average $D_{22}$ began to increase rapidly, and (iii) the moment of rupture are presented (see Figs. 8 (a4), (b4) and (c4)). Prior to the element deletion, damage was concentrated around the middle part of the RVE, related to larger effective normal stress in the tension direction ( $\left.\tilde{\sigma}_{22}\right)$ as shown in Figs. 9 (a1), (b1) and (c1). According to Eq. (16), all components of the effective stress contribute to $D_{22}$; however, the influence of $\tilde{\sigma}_{22}$ was larger due to the uniaxial tension state in the y-direction (corresponds to the 2 direction). Observed inhomogeneous stress distribution of $\tilde{\sigma}_{22}$ was due to the differences in grain orientation in the RVE. Element deletion was observed to occur in the stiffest grain which was spatially located at the RVE centre (coincidentally). When $D_{22}$ (the largest damage component) of an element reached or exceeded $D_{c}=0.4$, the element would be deleted, releasing the local stress concentration, and the stress was transferred to other stiff (in the y-direction) grains (shown in Figs. 9 (a2), (b2) and (c2)). As a result, element deletion was not limited to the middle part of the RVE, as shown in Figs. 9 (a3), (b3) and (c3). As the number of elements reduced gradually with increasing damage, implying creation of internal detects (microvoids) inside the material, the global material stiffness reduced. Therefore, material deformation became easier, accelerating damage accumulation and further deletion of elements. Strain was observed to increase rapidly, indicating the beginning of tertiary creep. 

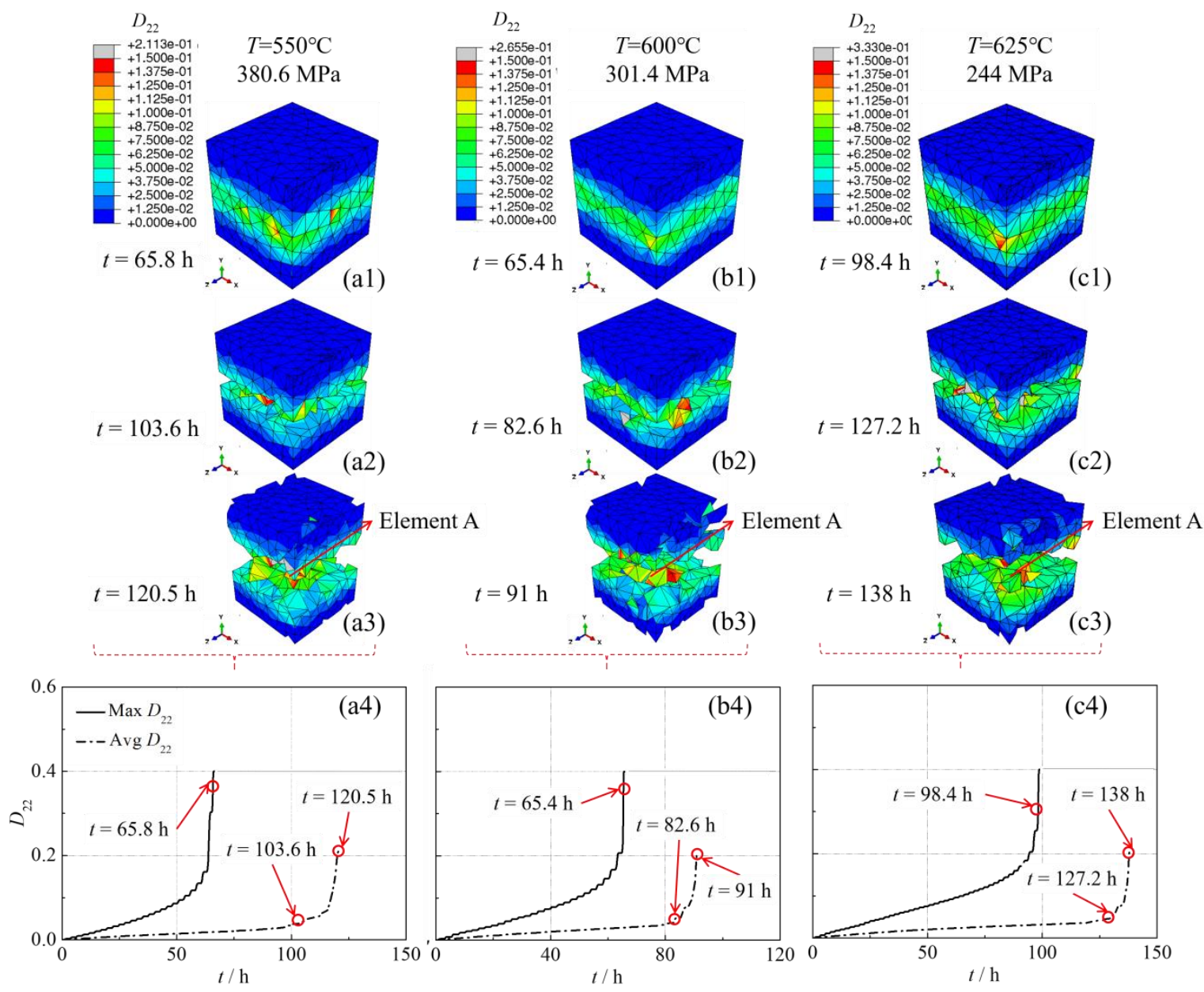

Fig. 8 Evolution of damage component $D_{22}$ at stress of $380.6 \mathrm{MPa}$ at $550^{\circ} \mathrm{C}((\mathrm{a} 1)-(\mathrm{a} 4)), 301.4 \mathrm{MPa}$ at $600^{\circ} \mathrm{C}((\mathrm{b} 1)-(\mathrm{b} 4))$ and $244 \mathrm{MPa}$ at $625^{\circ} \mathrm{C}((\mathrm{c} 1)-(\mathrm{c} 4))$.
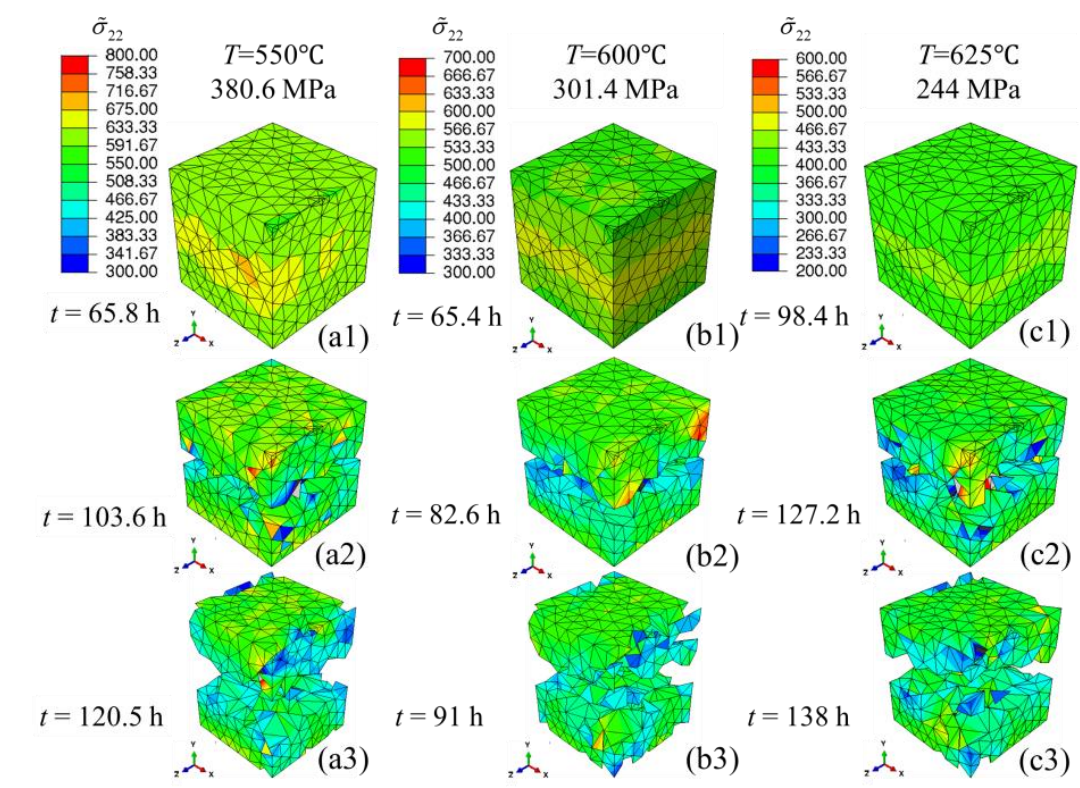

(c1)

Fig. 9 Evolution of effective stress component $\tilde{\sigma}_{22}$ at stress of $380.6 \mathrm{MPa}$ at $550^{\circ} \mathrm{C}((\mathrm{a} 1)-(\mathrm{a} 3)), 301.4$ $\mathrm{MPa}$ at $600^{\circ} \mathrm{C}((\mathrm{b} 1)-(\mathrm{b} 3))$ and $244 \mathrm{MPa}$ at $\left.625^{\circ} \mathrm{C}(\mathrm{c} 1)-(\mathrm{c} 3)\right)$. 
The temporal evolution of maximum and average magnitudes of $D_{22}$ are shown in Figs. 8 (a4), (b4) and (c4). In general, the curves for the three loading conditions show similarity. The rate of increase of maximum $D_{22}$ was significantly larger than that of average $D_{22}$ at the early stage of the creep process. With the deletion of the first element (which corresponds to the element with maximum $D_{22}$ ), an internal defect in the RVE was generated, affecting the surrounding elements of the simulated domain. At the end of each loading condition, the average $D_{22}$ reached a value of $\sim 0.2$. By inspection, the element $\mathrm{A}$ in the FE mesh (Figs. 8 (a3), (b3) and (c3)) was on the final fracture surfaces and had the largest value of $D_{22}$ at the end of the three loading processes. This particular element was found to be representative of the elements on the final fracture surfaces; thus, it was selected for an in-depth analysis of slip systems, to further assess the creep deformation process at the micro-scale.

\subsection{Evolution of slip systems}

In the CP model, the resolved shear stress $\left(\tau^{(\alpha)}\right)$ plays a decisive role in the development of slip strain (inelastic strain) (Eq. (6)). It was observed that the contribution of secondary slip systems to the overall slip strain was considerably lower; thus, only the contribution from the active main slip systems (MSSs) at the chosen element A is shown in Figs. 10 (a1), (a2) and (a3), along with the damage curves $\left(D_{22}\right)$ for the element (Figs. 10 (b1), (b2) and (b3)). The eight MSSs are detailed in Table 3. The evolution profile of $D_{22}$ in the selected element shows similarity with the global $D_{22}$ profile (Figs. 8 (a4), (b4) and (c4)). At each loading condition, $\tau^{(\alpha)}$ in the eight active MSSs may be roughly divided into four groups, with each group showing (almost) identical $\tau^{(\alpha)}$ values. For each MSS, $\tau^{(\alpha)}$ increased gradually over time, especially for MSSs 1, 6, 7, 11 with larger $\tau^{(\alpha)}$ (Figs. 10 (a1), (a2) and (a3)). At stress of $380.6 \mathrm{MPa}$ at $550^{\circ} \mathrm{C}, D_{22}$ increased rapidly at around $t=100 \mathrm{~h}$, accompanied by a rapid increase in $\tau^{(\alpha)}$. This evolution profile was similar to the other two loading conditions. With a growth in $D_{22}$, the effective stress $\tilde{\boldsymbol{\sigma}}$ increased, contributing to the rise in $\tau^{(\alpha)}$ (Eq. (12)). In addition, due to the reduction of the external stress level, the magnitude of $\tau^{(\alpha)}$ in each MSS reduced gradually from the first loading condition (at $380.6 \mathrm{MPa}$ at $550^{\circ} \mathrm{C}$ ) to the final test case (at $244 \mathrm{MPa}$ at $625^{\circ} \mathrm{C}$ ). However, the magnitude of $D_{22}$ did not vary greatly amongst the three loading conditions. This implies that at a higher temperature (from $550^{\circ} \mathrm{C}$ to $625^{\circ} \mathrm{C}$ ), accumulation of damage was easier, with increase of propensity for creep failure, even though the stress level and the slip's driving force $\left(\tau^{(\alpha)}\right)$ were lower. 


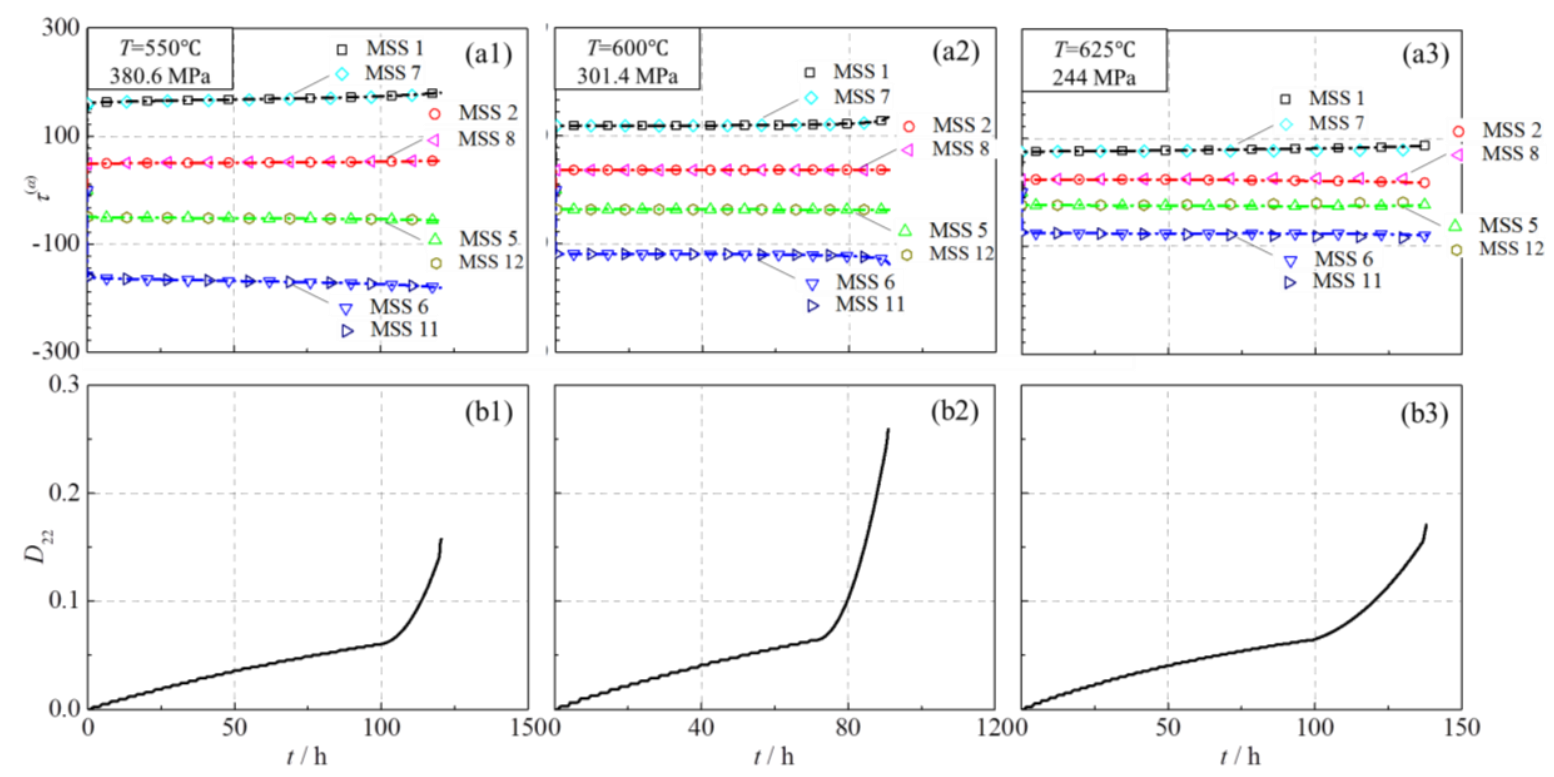

Fig. 10 Evolution of resolved shear stress $\left(\tau^{(\alpha)}\right)$ of MSSs and damage curves $\left(D_{22}\right)$ at element A at stress of $380.6 \mathrm{MPa}$ at $550^{\circ} \mathrm{C}((\mathrm{a} 1)$ and $(\mathrm{b} 1)), 301.4 \mathrm{MPa}$ at $600^{\circ} \mathrm{C}((\mathrm{a} 2)$ and $(\mathrm{b} 2))$ and $244 \mathrm{MPa}$ at $625^{\circ} \mathrm{C}$ ((a3) and (b3)).

Table 3 Active MSSs.

\begin{tabular}{c|c|c}
\hline MSSs & Slip plane & Slip direction \\
\hline 1 & $(011)$ & {$[1 \overline{1} 1]$} \\
\hline 2 & $(011)$ & {$[11 \overline{1}]$} \\
\hline 5 & $(110)$ & {$[\overline{1} 11]$} \\
\hline 6 & $(110)$ & {$[1 \overline{1} 1]$} \\
\hline 7 & $(0 \overline{1} 1)$ & {$[111]$} \\
\hline 8 & $(0 \overline{1} 1)$ & {$[\overline{1} 11]$} \\
\hline 11 & $(\overline{1} 10)$ & {$[111]$} \\
\hline 12 & $(\overline{1} 10)$ & {$[11 \overline{1}]$} \\
\hline
\end{tabular}

Additionally, the evolution of slip strain $\left(\gamma^{(\alpha)}\right)$ in MSSs for the chosen element A is presented in Fig. 11. According to the flow rule for $\dot{\gamma}^{(\alpha)}$ (Eq. (6)), due to a difference in $\tau^{(\alpha)}$ for the MSSs, $\gamma^{(\alpha)}$ was different, with a much larger variation (reaching several orders of magnitude) than that in $\tau^{(\alpha)}$. Therefore, the global inelastic strain of the element $\mathrm{A}$ was determined by slip strains from several dominant slip systems (MSSs 1, 6, 7, 11 here). For 
each MSS, $\gamma^{(\alpha)}$ increased at an accelerated pace at the tertiary creep stage, which agrees with the evolution of global strain with time (Fig. 5). Though the overall trend of $\gamma^{(\alpha)}$ evolution in all the MSSs is similar, the relative magnitudes of $\gamma^{(\alpha)}$ for the MSSs differ for the three loading conditions (e.g., $\gamma^{(\alpha)}$ in MSS 8 is approximately 1.8 times of that in MSS 2 at the stress of $380.6 \mathrm{MPa}$ at $550^{\circ} \mathrm{C}$, while 2.8 times at the stress of $244 \mathrm{MPa}$ at $625^{\circ} \mathrm{C}$, as shown in Figs. 11 (a2) and (c2)). For the single element on the final fracture surfaces, evolution of $\gamma^{(\alpha)}$ was not only related to the damage and effective stress state (with little difference for the three loading conditions), but also associated with the evolution of fracture surface. Once element deletion began on the fracture surface, the plastic slip was affected according to the orientation of slip systems. At different loading conditions, the state of element deletion on the fracture surfaces differed; thus, activation of some MSSs was different for the chosen element A, as shown in Figs. 11 (a2), (b2) and (c2).
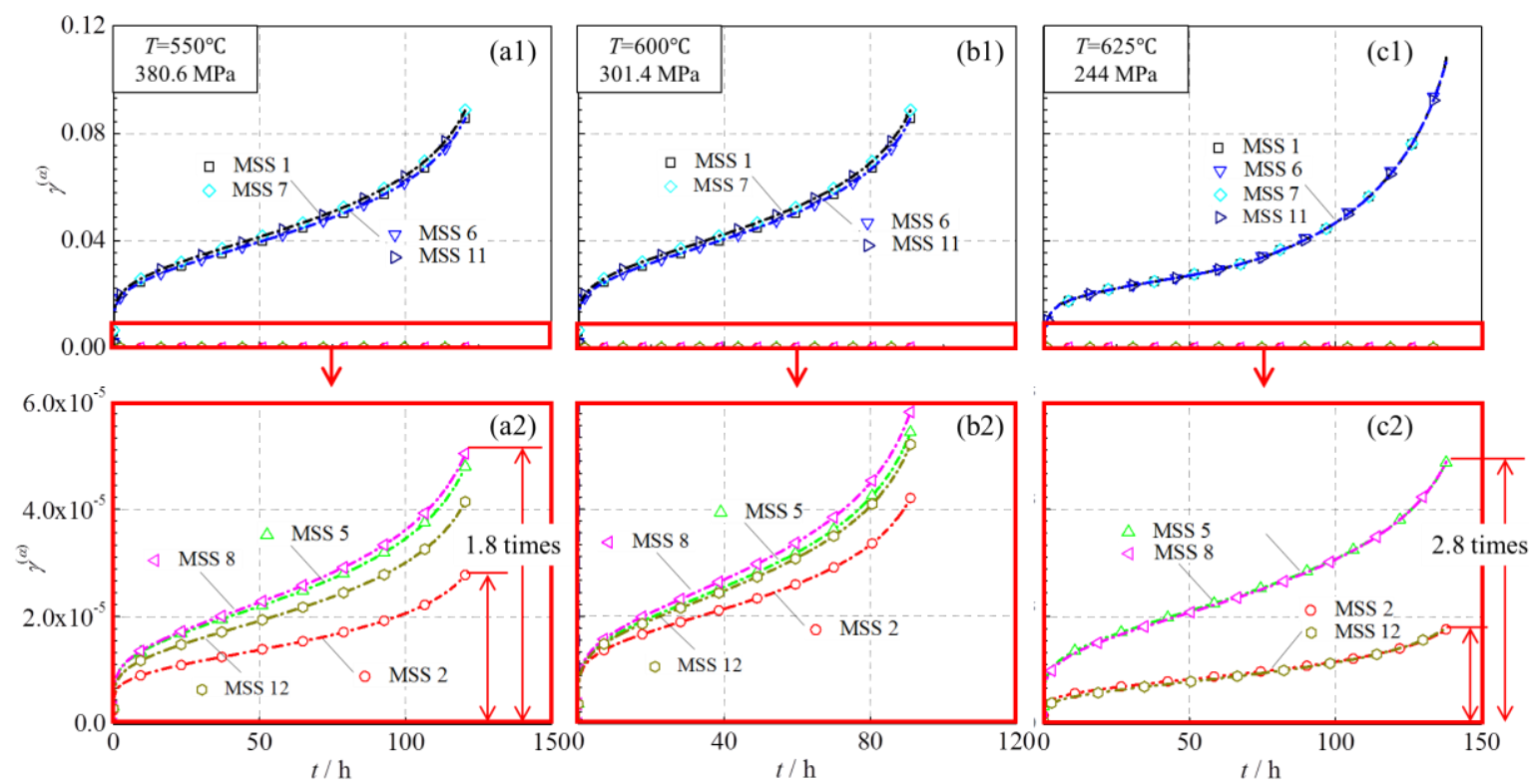

Fig. 11 Evolution of slip strain $\left(\gamma^{(\alpha)}\right)$ of active MSSs at element A: (a1) $380.6 \mathrm{MPa}$ at $550^{\circ} \mathrm{C}$; (b1) 301.4 MPa at $600^{\circ} \mathrm{C}$; (c1) $244 \mathrm{MPa}$ at $625^{\circ} \mathrm{C}((\mathrm{a} 2),(\mathrm{b} 2)$ and (c2) are zoomed-in views of respective boxes in (a1), (b1) and (c1)). 


\section{Conclusions}

In this paper, a coupled model of $\mathrm{CP}$ and $\mathrm{CDM}$ was proposed and implemented numerically for complete creep assessment of Cr-based steel. The CP theory was used to capture the anisotropic stress-strain response of the studied material due to the effect of grain morphology and crystallographic orientation. A second-order symmetric tensor was used to describe the anisotropic creep damage response, which affected both isotropic hardening and slip strain of slip systems. Uniaxial creep experiments at temperatures of $550^{\circ} \mathrm{C}, 600^{\circ} \mathrm{C}$, and $625^{\circ} \mathrm{C}$ at different stress levels were used for the calibration of CDM parameters. A series of numerical simulations were performed based on a developed polycrystalline RVE to demonstrate the capability of the developed coupled model for creep assessment. The main conclusions from this study are as follows:

1. The strain curves simulated based on the suggested numerical scheme and measured experimental results were in a fairly good agreement, illustrating the predictive capability of the coupled model.

2. The developed model can capture the underlying plastic anisotropy as well as the anisotropic damage evolution associated with the specific loading condition.

3. Analysis of slip variables revealed that at a higher temperature, damage accumulated with ease leading to creep failure, even with smaller resolved shear stresses. The global level of inelastic strain of the failed element was determined by slip strain from several dominant slip systems.

In this study, we consider a uniaxial tension loading condition for creep modelling, corresponding to the experimental condition. For a multiaxial loading condition, the coupled model is expected to predict the creep response accurately as it utilises an anisotropic damage variable. This will be investigated in the future. 


\section{ACKNOWLEDGEMENT}

The authors greatly acknowledge the financial support from the China Scholarship Council. AR acknowledges funding from the Engineering and Physical Sciences Research Council (UK) through grant EP/P027555/1, $\mathrm{H}^{2}$ Manufacturing.

\section{REFERENCES}

Abaqus, n.d. Abaqus 6.14 Documentation IV.

Anahid, M., Samal, M.K., Ghosh, S., 2011. Dwell fatigue crack nucleation model based on crystal plasticity finite element simulations of polycrystalline titanium alloys. J. Mech. Phys. Solids 59, 2157-2176. https://doi.org/10.1016/j.jmps.2011.05.003

Busso, E.P., Meissonnier, F.T., O’Dowd, N.P., 2000. Gradient-dependent deformation of twophase single crystals. J. Mech. Phys. Solids 48, 2333-2361. https://doi.org/10.1016/S0022-5096(00)00006-5

Cui, L., Wang, P., 2014. Two lifetime estimation models for steam turbine components under thermomechanical creep-fatigue loading. Int. J. Fatigue 59, 129-136. https://doi.org/10.1016/j.ijfatigue.2013.09.007

Dong, J., He, Y., Kim, M., Shin, K., 2013. Effect of creep stress on the microstructure of 9$12 \% \mathrm{Cr}$ steel for rotor materials. Microsc. Microanal. 19, 95-98. https://doi.org/10.1017/S1431927613012415

Ekh, M., Lillbacka, R., Runesson, K., 2004. A model framework for anisotropic damage coupled to crystal (visco)plasticity. Int. J. Plast. 20, 2143-2159. https://doi.org/10.1016/j.ijplas.2004.04.007

Feng, L., Zhang, G., Zhang, K.S., Xu, J.Q., Yu, H.D., 2004. On analysis of the elastoviscoplastic response of single crystals with anisotropic damage: Constitutive modelling and computational aspects. Int. J. Numer. Methods Eng. 61, 406-432. https://doi.org/10.1002/nme.1072

Golden, B.J., Li, D.F., O’Dowd, N.P., Tiernan, P., 2014. Microstructural modeling of P91 martensitic steel under uniaxial loading conditions. J. Press. Vessel Technol. 136, 021404. https://doi.org/10.1115/1.4026028

Hu, P., Liu, Y., Zhu, Y., Ying, L., 2016. Crystal plasticity extended models based on thermal mechanism and damage functions: Application to multiscale modeling of aluminum alloy tensile behavior. Int. J. Plast. 86, 1-25. https://doi.org/10.1016/j.ijplas.2016.07.001 
Huang, Y., 1991. A user-material subroutine incorporating single crystal plasticity in the abaqus finite element program. Harvard Univ.

Jiang, J., Wang, W., Zhao, N., Wang, P., Liu, Y., Jiang, P., 2015. Application of a creepdamage constitutive model for the rotor of a $1000 \mathrm{MW}$ ultrasupercritical steam turbine. J. Eng. Gas Turbines Power 138, 022606. https://doi.org/10.1115/1.4031323

JianPing, J., Guang, M., Yi, S., SongBo, X., 2003. An effective continuum damage mechanics model for creep-fatigue life assessment of a steam turbine rotor. Int. J. Press. Vessel. Pip. 80, 389-396. https://doi.org/10.1016/S0308-0161(03)00070-X

Jing, J., Meng, G., Sun, Y., Xia, S., 2003. An effective continuum damage mechanics model for creep-fatigue life assessment of a steam turbine rotor. Int. J. Press. Vessel. Pip. 80, 389-396. https://doi.org/10.1016/S0308-0161(03)00070-X

Kachanov, L.M., 1958. Time of the rupture process under creep conditions, Izv. Akad. Nauk SSR Otd Tech Nauk 8, 26-31.

Kalnaus, S., Jiang, Y., 2006. Fatigue life prediction of copper single crystals using a critical plane approach. Eng. Fract. Mech. 73, 684-696. https://doi.org/10.1016/j.engfracmech.2005.11.003

Kovač, M., Cizelj, L., 2005. Modeling elasto-plastic behavior of polycrystalline grain structure of steels at mesoscopic level. Nucl. Eng. Des. 235, 1939-1950. https://doi.org/10.1016/j.nucengdes.2005.05.009

Lemaitre, J., Chaboche, J.-L., 1994. Mechanics of solid materials. Cambridge University Press.

Li, D.F., Barrett, R.A., O’Donoghue, P.E., O’Dowd, N.P., Leen, S.B., 2017. A multi-scale crystal plasticity model for cyclic plasticity and low-cycle fatigue in a precipitatestrengthened steel at elevated temperature. J. Mech. Phys. Solids 101, 44-62. https://doi.org/10.1016/j.jmps.2016.12.010

Luo, C., Chattopadhyay, A., 2011. Prediction of fatigue crack initial stage based on a multiscale damage criterion. Int. J. Fatigue 33, 403-413. https://doi.org/10.1016/j.ijfatigue.2010.09.014

Luo, C., Wei, J., Garcia, M.P., Chattopadhyay, A., Peralta, P., 2009. Fatigue damage prediction in metallic materials based on multiscale modeling 47. AIAA J. 47, 25672576. https://doi.org/10.2514/1.39559

Meissonnier, F.T., Busso, E.P., O’Dowd, N.P., 2001. Finite element implementation of a generalised non-local rate-dependent crystallographic formulation for finite strains. Int. 
J. Plast. 17, 601-640. https://doi.org/10.1016/S0749-6419(00)00064-4

Potirniche, G.P., Horstemeyer, M.F., Ling, X.W., 2007. An internal state variable damage model in crystal plasticity. Mech. Mater. 39, 941-952.

https://doi.org/10.1016/j.mechmat.2007.04.004

Qi, W., Bertram, A., 1999. Anisotropic continuum damage modeling for single crystals at high temperatures. Int. J. Plast. 15, 1197-1215. https://doi.org/doi: DOI: 10.1016/S07496419(99)00035-2

Qi, W., Bertram, A., 1998. Damage modeling of the single crystal superalloy SRR99 under monotonous creep. Comput. Mater. Sci. 13, 132-141. https://doi.org/10.1016/S09270256(98)00053-6

Quey, R., Dawson, P.R., Barbe, F., 2011. Large-scale 3D random polycrystals for the finite element method: Generation, meshing and remeshing. Comput. Methods Appl. Mech. Eng. 200, 1729-1745. https://doi.org/10.1016/j.cma.2011.01.002

Rabotnov, Y.N., 1969. Creep rupture, in: Applied Mechanics. Springer, pp. 342-349.

Roters, F., Eisenlohr, P., Hantcherli, L., Tjahjanto, D.D., Bieler, T.R., Raabe, D., 2010. Overview of constitutive laws, kinematics, homogenization and multiscale methods in crystal plasticity finite-element modeling: Theory, experiments, applications. Acta Mater. 58, 1152-1211. https://doi.org/10.1016/j.actamat.2009.10.058

Shi, D.Q., Yang, X.G., 2004. Application of the time-hardening creep law coupling damage. J. Aerosp. Power 19, 12-16.

Sinha, S., Ghosh, S., 2006. Modeling cyclic ratcheting based fatigue life of HSLA steels using crystal plasticity FEM simulations and experiments. Int. J. Fatigue 28, 1690-1704. https://doi.org/10.1016/j.ijfatigue.2006.01.008

Wang, W., 2014. Analysis of Multi-Axial Creep-Fatigue Damage on an Outer Cylinder of a 1000 MW Supercritical Steam Turbine. J. Eng. Gas Turbines Power 136, 112504. https://doi.org/10.1115/1.4027634

Wang, W.Z., Buhl, P., Klenk, A., 2015. A unified viscoplastic constitutive model with damage for multi-axial creep-fatigue loading. Int. J. Damage Mech. 24, 363-382. https://doi.org/10.1177/1056789514537007

Wichtmann, A., n.d. Evaluation of creep damage due to void growth under triaxial stress states in the design of steam turbine components. JSME Int. J. Series A Solid Mech. Mater. Eng 45, 72-76.

Xu, H.Z.M., 2007. Research on creep rupture of fastening bolts of ultra-supercritical steam 
turbine cylinder case at high temperature. Proc. Chinese Soc. Electr. Eng. 2, 2007.

Yaso, M., Hayashi, S., Morito, S., Ohba, T., Kubota, K., Murakami, K., 2009. Characteristics of retained austenite in quenched high C-high Cr alloy steels. Mater. Trans. 50, 275-279. https://doi.org/10.2320/matertrans.MRA2008161

Zhang, K.S., Ju, J.W., Li, Z., Bai, Y.L., Brocks, W., 2015. Micromechanics based fatigue life prediction of a polycrystalline metal applying crystal plasticity. Mech. Mater. 85, 16-37. https://doi.org/10.1016/j.mechmat.2015.01.020

Zhang, K.S., Shi, Y.K., Ju, J.W., 2013. Grain-level statistical plasticity analysis on strain cycle fatigue of a FCC metal. Mech. Mater. 64, 76-90. https://doi.org/10.1016/j.mechmat.2013.05.001

Zhao; Wang; Liu, n.d. Startup fatigue behavior study of a steam turbine rotor based on crystal plasticity with macroscale phenomenological constitutive models. (unpubl. results). 